\title{
A Self-Critique of Self-Organized Criticality in Astrophysics
}

\author{
Markus J. Aschwanden \\ Lockheed Martin Solar and Astrophysics Laboratory, \\ Bldg. 252, Org A021S, 3251 Hanover St., Palo Alto, CA 94304, USA \\ email: aschwanden@lmsal.com
}

\begin{abstract}
The concept of "self-organized criticality" (SOC) was originally proposed as an explanation of 1/f-noise by Bak, Tang, and Wiesenfeld (1987), but turned out to have a far broader significance for scale-free nonlinear energy dissipation processes occurring in the entire universe. Over the last 30 years, an inspiring cross-fertilization from complexity theory to solar and astrophysics took place, where the SOC concept was initially applied to solar flares, stellar flares, and magnetospheric substorms, and later extended to the radiation belt, the heliosphere, lunar craters, the asteroid belt, the Saturn ring, pulsar glitches, soft X-ray repeaters, blazars, black-hole objects, cosmic rays, and boson clouds. The application of SOC concepts has been performed by numerical cellular automaton simulations, by analytical calculations of statistical (powerlaw-like) distributions based on physical scaling laws, and by observational tests of theoretically predicted size distributions and waiting time distributions. Attempts have been undertaken to import physical models into numerical SOC toy models. The novel applications stimulated also vigorous debates about the discrimination between SOC-related and non-SOC processes, such as phase transitions, turbulence, random-walk diffusion, percolation, branching processes, network theory, chaos theory, fractality, multi-scale, and other complexity phenomena. We review SOC models applied to astrophysical observations, attempt to describe what physics can be captured by SOC models, and offer a critique of weaknesses and strengths in existing SOC models.
\end{abstract}

Keywords. Self-organized criticality, solar physics, astrophysics

The original definition of the term self-organized criticality (SOC) was inspired by a numerical lattice simulation of a dynamical system with spatially complex patterns, mimicking avalanches of a sandpile, which became the BTW model (Bak, Tang, and Wiesenfeld 1987), and demonstrated that:

- Dynamical systems with extended spatial degrees of freedom naturally evolve into self-organized critical structures of states which are barely stable. Flicker noise, or $1 / f$ noise, can be identified with the dynamics of the critical state. This picture also yields insight into the origin of fractal objects. (Bak et al. 1987)

In this first seminal paper, the authors had already fractal structures like cosmic strings, mountain landscapes, and coastal lines as potential applications in mind and concluded: We believe that the new concept of self-organized criticality can be taken much further and might be the underlying concept of dissipative systems with extended degrees of freedom (Bak et al. 1987). In this spirit, the application of the SOC concept has been broadened substantially over the last 25 years. If we read a recent definition of SOC, we find:

- Self-organized criticality is regarded as scale invariance without external tuning of a control parameter, but with all the features of the critical point of an ordinary phase transition, in particular long range (algebraic) spatiotemporal correlations (Pruessner 2012). 

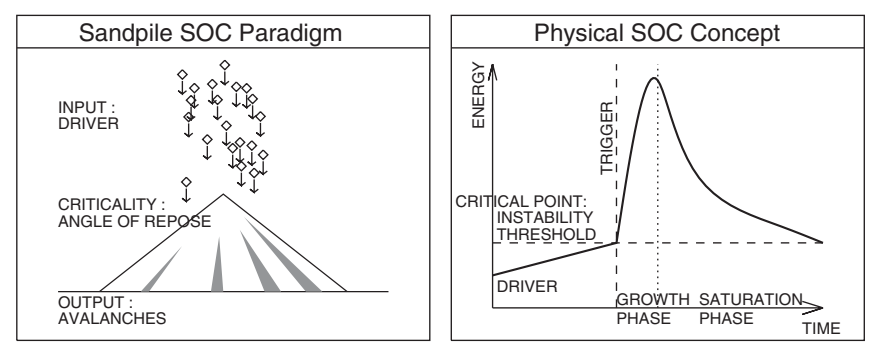

Figure 1. Left: The original sandpile SOC paradigm, consisting of the (input) driver, the self-organized criticality mechanism (self-tuning angle of repose), and the (output) avalanches. Right: In a physical SOC concept, the driver is a slow and continuous energy input rate, the criticality mechanism is replaced by a critical point in form of an instability threshold, where an avalanche is triggered, usually consisting of a nonlinear growth phase and a subsequent saturation phase.

In the same vein, it is stated in the original paper of the SOC creators: The criticality in our theory is fundamentally different from the critical point at phase transitions in equilibrium statistical mechanics which can be reached by tuning of a parameter, for instance the temperature (Bak et al. 1987). The aspect of self-tuning in SOC systems is the most crucial difference to (second-order) phase transitions, where fine-tuning is necessary and is not automatically arranged by nature. The implications and theoretical details of this peculiar feature are discussed in Watkins et al. (2014). However, whenever there is a threshold for instabilities, the threshold value itself could be called a "critical point" that decides whether an instability, also called a nonlinear energy dissipation event, or avalanche, happens or not. Over the past 25 years, a lot of applications of the SOC concept have been made to slowly-driven systems with a critical threshold, especially in solar and astrophysics, as reviewed here. A more pragmatic and physics-based definition of a SOC system is:

- $S O C$ is a critical state of a nonlinear energy dissipation system that is slowly and continuously driven towards a critical value of a system-wide instability threshold, producing scale-free, fractal-diffusive, and intermittent avalanches with powerlaw-like size distributions (Aschwanden 2014).

With this definition we broaden the meaning of the term "criticality" to a more general meaning of a "critical point", which includes almost any nonlinear system with a (global) instability threshold (Fig. 1). In addition, a SOC system has to be self-organizing or self-tuning without external control parameter, which is accomplished by a slow and continuous driver, which brings the system back to the critical point after each avalanche. Thus, we can say that a SOC system has energy balance between the slowly-driven input and the (spontaneous) avalanching output, and thus energy is conserved in the system (in the time average).

\section{References}

Aschwanden, M. J. 2014, ApJ, 782, 54

Aschwanden, M. J., Crosby, N., Dimitropoulou, M., Georgoulis, M. K., et al. 2015, Space Science Reviews (online first), DOI 10.1007/s11214-014-0054-6

Bak, P., Tang, C., \& Wiesenfeld, K. 1987, PhRvL 59/4, 381

Pruessner, G. 2012, Self-organised criticality. Theory, models and characterisation, ISBN 9780521853354, Cambridge University Press, Cambridge

Watkins, N. W., Pruessner, G., Chapman, S. C., Crosby, N. B., and Jensen, H.J. 2015 Space Science Reviews (online first), DOI 10.1007/s11214-015-0155-x 